\title{
Lixivaptan - an evidence-based review of its clinical potential in the treatment of hyponatremia
}

This article was published in the following Dove Press journal:

Core Evidence

10 July 2013

Number of times this article has been viewed

\section{Brendan T Bowman}

Mitchell H Rosner

Division of Nephrology, University of Virginia Health System,

Charlottesville, VA, USA
Correspondence: Mitchell H Rosner Division of Nephrology, Box 800133 HSC, University of Virginia Health System, Charlottesville, VA 22908, USA

Tel + I 4349826999

Fax +l 4349245848

Emailmhr9r@virginia.edu
Abstract: Hyponatremia is the most common electrolyte abnormality seen in clinical practice. Most cases of euvolemic or hypervolemic hyponatremia involve arginine vasopressin (AVP). AVP leads to a concentrated urine and negative free water clearance. Given this primary role of AVP, antagonizing its effect through blockade of its receptor in the distal tubule is an attractive therapeutic target. Lixivaptan is a newer, non-peptide, vasopressin type 2 receptor antagonist. Recent studies have demonstrated efficacy. This review summarizes the clinical pharmacology and data for this new agent.

Keywords: vasopressin, hyponatremia, heart failure, lixivaptan, therapy, outcomes

Core evidence clinical impact summary for Lixivaptan in hyponatremia

\begin{tabular}{|c|c|c|}
\hline $\begin{array}{l}\text { Outcome } \\
\text { measure }\end{array}$ & Evidence & Implications \\
\hline \multicolumn{3}{|c|}{ Disease-oriented evidence } \\
\hline $\begin{array}{l}\text { Increase in serum } \\
\text { sodium levels }\end{array}$ & Strong & $\begin{array}{l}\text { Lixivaptan reliably raises serum } \\
\text { Na levels in euvolemic and } \\
\text { hypervolemic hyponatremia due to } \\
\text { SIADH and congestive heart failure }\end{array}$ \\
\hline $\begin{array}{l}\text { Increase in days } \\
\text { alive out of } \\
\text { hospital for heart } \\
\text { failure patients }\end{array}$ & Absent & $\begin{array}{l}\text { The BALANCE trial was unable to } \\
\text { show an increase in days alive and } \\
\text { out of the hospital measure for } \\
\text { heart failure patients }\end{array}$ \\
\hline \multicolumn{3}{|c|}{ Patient-oriented evidence } \\
\hline $\begin{array}{l}\text { Reduction in } \\
\text { need for fluid } \\
\text { restriction }\end{array}$ & Limited & $\begin{array}{l}\text { Trial results suggest patients may } \\
\text { be able to achieve normal serum } \\
\text { sodium with less need for fluid } \\
\text { restriction }\end{array}$ \\
\hline $\begin{array}{l}\text { Improvement } \\
\text { in cognitive } \\
\text { symptoms of } \\
\text { hyponatremia }\end{array}$ & Absent & $\begin{array}{l}\text { Use of the lixivaptan did not yield } \\
\text { improvements in the Trail Making } \\
\text { Test Part B versus placebo }\end{array}$ \\
\hline $\begin{array}{l}\text { Improvement } \\
\text { in symptoms } \\
\text { attributable to } \\
\text { hyponatremia }\end{array}$ & Absent & $\begin{array}{l}\text { Improvement in serum sodium } \\
\text { has been used as a surrogate } \\
\text { for symptom improvement; but } \\
\text { direct evaluation of symptom } \\
\text { improvement remains to be studied }\end{array}$ \\
\hline \multicolumn{3}{|c|}{ Economic evidence } \\
\hline $\begin{array}{l}\text { Cost-effective } \\
\text { in treatment of } \\
\text { hyponatremia }\end{array}$ & Absent & $\begin{array}{l}\text { No data - further studies are } \\
\text { needed to determine if potential } \\
\text { savings from theoretical decreased } \\
\text { admissions could justify pricing }\end{array}$ \\
\hline
\end{tabular}




\section{Overview of hyponatremia}

Hyponatremia is the most common electrolyte abnormality encountered in both inpatient and ambulatory settings. ${ }^{1}$ Prevalence is highest among hospitalized patients and elderly ambulatory patients, approaching $30 \%$ and $12 \%$, respectively. ${ }^{2,3}$ Traditionally, hyponatremia has been defined as a serum sodium less than $136 \mathrm{mmol} / \mathrm{L}$, though there is variation between studies, which partially explains the variability in prevalence rates across some studies. ${ }^{4}$ Hyponatremia has been associated with numerous poor outcomes, including cognitive impairment, increased fracture rates, impairment in gait stability, and increased mortality. ${ }^{5-7}$ Even "mild" hyponatremia (often defined as serum sodium 130-135 mmol/L) has been shown to be associated with significant mortality and morbidity. ${ }^{7}$ Hyponatremia also imposes a significant economic burden, raising costs $40 \%-50 \%$ versus similar "normonatremic" patients. ${ }^{8}$

While hyponatremia is diagnosed by an abnormally low concentration of the serum sodium, physiologically, this represents a problem of water imbalance: an elevated ratio of total body water to total body sodium. Traditionally, a patient's volume status has suggested etiology and dictated treatment. Hyponatremia is then generally categorized into three groups: hypervolemic, euvolemic, and hypovolemic. ${ }^{4}$ While hypovolemic hyponatremia responds well to isotonic fluid administration, hypervolemic and euvolemic hyponatremia can be difficult to effectively treat using currently available therapies (eg, fluid restriction and diuretics). Part of this difficulty in management is related to the role of vasopressin in hyponatremia, which traditional therapies did not effectively target.

In normal physiology, vasopressin is released from the pituitary gland primarily in response to increases in serum osmolality sensed via osmoreceptors in the hypothalamus. In addition, vasopressin release is also stimulated by volume depletion. ${ }^{9}$ Vasopressin receptors exist in varying forms found throughout the body. There are three known vasopressin receptor types: V1a, V1b (V3), and V2. ${ }^{10,11}$ The $\mathrm{V} 1$ a receptor form is found in vascular smooth muscle and the myocardium, V1b (V3) is found in the anterior pituitary,

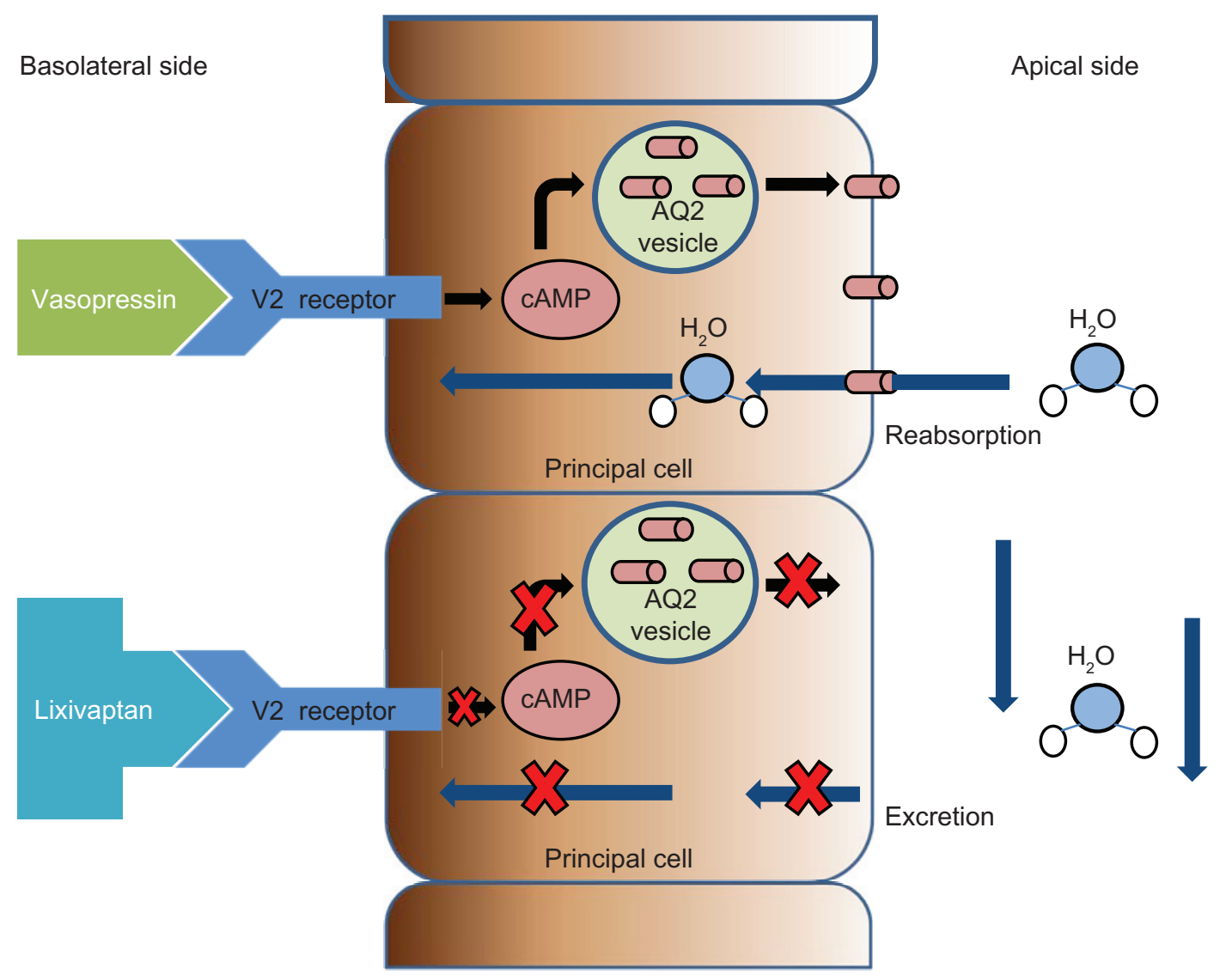

Figure I Binding of vasopressin on the basolateral side of the principal cell activates a Gs-coupled protein which activates adenylyl cyclase (not pictured), increasing cyclic AMP concentrations. AQ2 is released from preformed vesicles and inserts in the apical membrane. Blocking of the V2 receptor leads to lack of AQ2 channel insertion and eventually a free water diuresis.

Abbreviations: cAMP, cyclic adenosine monophosphate; AQ2, aquaporin-2. 
and the V2 form predominates in the kidney, notably in the collecting duct. There, binding of vasopressin on the basolateral side of the principal cell activates a $\mathrm{G}_{\mathrm{s}}$-coupled protein which, in turn, activates adenylyl cyclase, increasing cyclic adenosine monophosphate concentrations. The net effect is release of aquaporin-2 from preformed vesicles with insertion of these water channels into the luminal side of the collecting duct (Figure 1). A path for free water reabsorption is created down an osmolar gradient, which is maintained in the medullary interstitium of the kidney. In this manner, vasopressin plays a key role in maintaining both plasma volume as well as plasma osmolality. ${ }^{12}$ Thus, under the influence of vasopressin, free water reabsorption in the distal nephron is enhanced, leading to a concentrated, low volume urine (and return of free water to the circulation). In the absence of vasopressin, free water excretion is increased, leading to a high volume, dilute urine.

In hypervolemic and euvolemic hyponatremia, the mechanism of vasopressin release is altered (ie, nonosmotically controlled), but the end result is the same laboratory abnormality - a diluted serum sodium. In hypervolemic hyponatremia, the extra cellular water is expanded but effective circulating volume is reduced. This leads to increased sodium and water retention via various mechanisms, including increased vasopressin action. Sodium retention results largely from activation of the renin-angiotensin-aldosterone system; however, persistent vasopressin stimulation from decreased effective circulating volume leads to free water retention and eventual hyponatremia. This stimulus for vasopressin release can be strong enough to overcome the usually vasopressin-inhibitory decrease in plasma osmolality and result in excessive water retention. ${ }^{9}$ This scenario contrasts with the euvolemic hyponatremia patient where the syndrome of inappropriate antidiuretic hormone (SIADH) predominates as the most common cause. ${ }^{13}$ In the case of SIADH, various intrinsic (pain, nausea, and malignancy) or extrinsic (medications) stimuli lead to release of vasopressin that is in excess of physiologic needs. The typical patient with SIADH has only mild volume increase, but the degree of hyponatremia may be quite profound. ${ }^{13,14}$

\section{Implications of hyponatremia}

Hyponatremia has a variety of clinical and economic implications on both patients and the health care system as a whole. Severe acute hyponatremia, defined variably in studies, but often as hyponatremia occurring in less than 24 hours and/or a serum sodium $<120 \mathrm{mmol} / \mathrm{L}$, has long been known to produce serious neurological complications due, in part, to acute cerebral edema and increased intracranial pressure. ${ }^{5,15}$ These may include profoundly altered mental status evidenced by decreased sensorium, seizure, coma, or even death. The pathophysiology has been attributed to rapid osmolar shifts leading to an overall influx of free water across the blood-brain barrier, resulting in cerebral edema. With little room to expand, cerebral edema may lead to herniation and subsequent death. ${ }^{16}$

More recently, mild to moderate chronic hyponatremia, often termed "asymptomatic hyponatremia," has been demonstrated to have a number of clinically important associations. These may include increased rates of fractures, subclinical cognitive changes, and increased mortality. $6,7,17$ This is particularly true in the elderly. Hoorn et al prospectively followed a cohort of over 5,200 subjects 55 years and older in Rotterdam, The Netherlands. ${ }^{6}$ They determined a prevalence of hyponatremia at $7.7 \%$, with a mean serum sodium of $133 \mathrm{mmol} / \mathrm{L}$. Among the hyponatremic group, even when matched for disability index, use of diuretics, and psychotropic medications, the rates of vertebral fractures were higher than their normonatremic counterparts, with a hazard ratio of 1.6.

Renneboog and colleagues also studied chronic, "asymptomatic hyponatremia" for evidence of attention deficits and increased falls in emergency department patients. ${ }^{17}$ In their study, 122 patients in total were evaluated for fall-related admissions. Compared with a control group, the chronic hyponatremia patients had a fall incidence of $21 \%$ versus approximately $5 \%$ in the matched controls. The investigators also tested 16 hyponatremic patients via standardized computer-based exams to evaluate attention deficits; both response time and accuracy were recorded. Hyponatremic patients consistently demonstrated statistically significant delays in response time and higher error rates than controls. The hyponatremic group was also compared with a group of healthy volunteers following alcohol ingestion (average blood alcohol content of $0.6 \mathrm{~g} / \mathrm{L}$ ). Even between these two groups, the hyponatremic patients performed significantly worse.

Verbalis and colleagues studied the association of hyponatremia and bone mineral density in both an animal model and a cross-sectional analysis of data from the Third National Health and Nutrition Examination Survey III (NHANES III). ${ }^{18}$ Evaluating NHANES data, they demonstrated increased rates of osteoporosis in patients with mild hyponatremia (mean $133 \mathrm{mmol} / \mathrm{L}$ ) when compared with an agematched cohort. The hyponatremic patients were 2.87 times more likely to meet criteria for osteoporosis of the femoral neck despite adjustment for variables known to affect bone 
mineralization such as vitamin D levels. While not demonstrating causation, the association of hyponatremia with increased rates of osteoporosis, falls, and fractures suggests a potential under-recognized clinical impact in the need for further studies.

\section{Mortality in hyponatremia}

Mortality due to cerebral edema, seizure, and herniation in severe acute hyponatremia has been described previously and is well known; ${ }^{15}$ however, more recent studies have established the mortality risk in previously presumed asymptomatic hyponatremia. In the study referenced previously by Hoorn, mild chronic hyponatremia was associated with a hazard ratio of 1.2 for all-cause mortality, despite adjustment for comorbidity. ${ }^{6}$ Wald and colleagues also evaluated hyponatremia and association with all-cause mortality. ${ }^{19}$ In their retrospective cohort study of over 50,000 hospital admissions, the authors evaluated "community-acquired hyponatremia" (patients admitted with preexisting hyponatremia), "hospital-aggravated hyponatremia" (preexisting hyponatremia that worsens in hospital) and "hospital-acquired hyponatremia" (development of hyponatremia after normal value at admission). In all three groups, hyponatremia remained a risk factor for in-hospital mortality. Mild hyponatremia in the community-acquired group demonstrated an odds ratio of 1.52 for in-hospital mortality, while the hospital-acquired hyponatremia group displayed a similar pattern, with an odds ratio of 1.61 in the mild group, rising to a troubling 14.39 in the severe group (defined as $<127 \mathrm{mEq} / \mathrm{L}$ ).

Despite adjusting for comorbidities and demographics, the question remains whether the mortality associated with hyponatremia reflects underlying disease severity or whether hyponatremia is a truly independent risk factor for mortality. Depending on the clinical situation, both scenarios can be true. For example, severe hyponatremia in edematous states such as cirrhosis and congestive heart failure can be viewed as a surrogate marker of disease progression. ${ }^{20,21}$ In these situations, worsening hyponatremia typically reflects progression to end-stage liver disease or decompensated heart failure - conditions with high mortality regardless of serum sodium. Attributing mortality risk to a particular level of serum sodium can be challenging and should be considered in the context of the individual patient.

\section{Economic impact of hyponatremia}

The presence of hyponatremia has a variety of clinical effects and adds complexity to both inpatient and outpatient management. Evaluating the economic impact in individual cases, and the health system in general, is challenging due to difficulty isolating costs directly attributable to hyponatremia versus those only associated with underlying diseases. For example, a patient may have end-stage liver disease with severe hyponatremia, necessitating prolonged inpatient admission and ICU (intensive care unit) care - regardless of the presence of hyponatremia. This can be contrasted with the case of postoperative hyponatremia where nausea, vomiting, or pain may trigger high vasopressin levels and an unanticipated prolonged stay. In the former, care is complicated, but not necessarily prolonged, whereas in the latter, care is both complicated and length of stay (LOS) increased. Despite these challenges, several retrospective studies have tried to evaluate costs via LOS, average cost increase per admission, and aggregate costs at the hospital and health system level. , $^{8,22-24}$

Several studies have evaluated LOS and cost increases per admission associated with hyponatremia. ${ }^{19,23,24}$ Zilberberg and colleagues reviewed over 190,000 admissions for a 1-year period from 2004 to 2005, comparing mortality, LOS, and incremental costs associated with hyponatremia. ${ }^{24}$ They found an average LOS increase of 1 day and overall cost increase of US\$2,289 per episode of care. Similarly, Callahan and colleagues found LOS increases of 2-3 days and median cost increases of $\sim$ US $\$ 1,400-U S \$ 3,000 .{ }^{23}$ Extrapolating their data to a national set, the investigators estimated a US\$1.1 billion additional cost to the US health care system. Shea et al evaluated annual costs of health plan members with hyponatremia versus normonatremic plan members and found roughly a $40 \%$ increase in expenditures on the hyponatremic group. ${ }^{8}$ The highest health system figure to date was provided by Boscoe and colleagues, estimating a range of US $\$ 1.6$ billion to US\$3.6 billion in additional direct costs alone. ${ }^{22}$ While all of these studies are retrospective, they suggest hyponatremia is associated with significant costs and economic impacts.

\section{Current therapies for hyponatremia}

Current therapies for euvolemic and hypervolemic hyponatremia (aside from treatment of underlying disease) can be considered to fall under three main strategies: reduction of solute-free fluid intake, increased solute intake, and increased solute free water restriction. Free water restriction is often the first strategy employed, and while this is tolerated in the short term, it is often limited by patient compliance, particularly for outpatients, and can be slow to take effect. ${ }^{25}$ Increased solute intake generally relies on sodium chloride tablet ingestion or hypertonic saline, which are also difficult to implement for outpatients. Urea as an oral preparation can be given to increase 
solute delivery to the distal tubule and obligate fluid loss; however, $15 \%$ of patients report unpleasant taste and it is not widely available in the US. ${ }^{26}$ In cases where fluid restriction and solute intake is unsuccessful, increased free water excretion can be attempted via several medications. Typically this takes the form of a loop diuretic, which inhibits the concentrating effect of vasopressin on the urine reducing the medullary concentration gradient. ${ }^{27}$ Both demeclocycline and lithium have been used to inhibit vasopressin activity and increase free water excretion; however, both have significant side-effect profiles (including renal toxicity) and are not routinely used..$^{25}$

In the US, there are currently two available (approved by the US Food and Drug Administration [FDA]) vasopressin antagonists: conivaptan (Astellas Pharma US, Northbrook IL, USA) and tolvaptan (Otsuka Pharmaceutical Development, Princeton NJ, USA and Commercialization, Inc, Princeton NJ, USA). Conivaptan is a parenterally administered mixed V1a and V2 receptor and has been available since 2004 for management of euvolemic and hypervolemic hyponatremia. Tolvaptan is an orally available V2 receptor-specific antagonist, also approved for euvolemic and hypervolemic hyponatremia. ${ }^{28,29}$

This class of drug, often referred to as "vaptans," mimic the binding site of vasopressin with similar affinity and without concomitant agonist activity. This leads to decreased insertion of aquaporin-2 channels and decreased water reabsorption in the collecting duct. ${ }^{30}$ The net effect is "aquaresis" or electrolyte free water excretion. Suggested applications for this drug class have included the treatment of hyponatremia, edematous states in general, and more recently, autosomal dominant polycystic kidney disease (ADPKD). ${ }^{31-33}$

Regulatory approval for the use of conivaptan in euvolemic and hypervolemic hyponatremia rests largely on a trial of 84 hospitalized patients with hyponatremia between 115 and $130 \mathrm{mEq} / \mathrm{L} .{ }^{34}$ Patients were administered an intravenous loading dose and subsequent daily infusion of either 40 or $80 \mathrm{mg}$ versus placebo. The 40 and $80 \mathrm{mg}$ groups attained mean sodium increases of 6.3 and $9.4 \mathrm{mEq} / \mathrm{L}$ respectively. A 2001study in heart failure patients demonstrated treatment with conivaptan was able to improve urinary output and decrease pulmonary capillary wedge pressure. ${ }^{32}$

The oral formulation of conivaptan has also been studied in two randomized controlled trials, showing statistically significant improvements in serum sodium. ${ }^{35,36}$ Due to potent inhibition of the cytochrome P450 system (CYP3A4) with the high potential for drug-drug interaction, the FDA has approved only the parenteral formulation.
Tolvaptan has been approved in the US since 2009 for the treatment of euvolemic and hypervolemic hyponatremia as an oral formulation. Approval was based on the Study of Ascending Levels of Tolvaptan (SALT) 1 and SALT 2 trials where a total of 448 patients with mild or marked hyponatremia were randomized to tolvaptan versus placebo, with statistically significant improvement in serum sodium at days 4 and $30 .{ }^{31}$ The benefits were not enduring however, and serum sodium declined to prior baseline with cessation of tolvaptan therapy. Tolvaptan has also been evaluated for treatment of heart failure symptoms. In a 2004 trial, Gheorghiade and colleagues demonstrated statistically significant improvements in weight loss versus placebo in patients admitted with congestive heart failure. ${ }^{37}$ A posthoc analysis of secondary endpoints suggested superior 60 -day mortality results for the tolvaptan group as well (5.4\% versus $8.7 \%$ mortality rate). A subsequent randomized controlled trial of over 4,000 patients admitted with heart failure evaluated all-cause and cardiovascular death in patients randomized to placebo versus tolvaptan $30 \mathrm{mg}$ daily. ${ }^{38}$ Unfortunately, the encouraging results of the previous heart failure trial were not replicated, and both all-cause and cardiovascular mortality did not differ between groups over a median follow-up of 9.9 months. More recently, tolvaptan has been studied in ADPKD in the Tolvaptan Efficacy and Safety in Management of Autosomal Dominant Polycystic Kidney Disease and its Outcomes (TEMPO) 3:4 trial. ${ }^{33}$ In this study, tolvaptan was shown to slow the increase in kidney volume associated with ADPKD over a 3-year period, and further trials are ongoing.

Despite the promise of vasopressin receptor antagonists, both currently available medications have not been without a down side. Conivaptan has been associated with a high rate of infusion site reactions, orthostatic hypotension, and is only available as an intravenous formulation. ${ }^{39}$ This effectively limits usage to inpatients only. The longer-term SALTWATER (Safety and Sodium Assessment of Long-term Tolvaptan with hyponatremia) trial, while generally showing good tolerability, did demonstrate a number of side effects to tolvaptan, including pollakiuria, polyuria, excessive thirst, and dry mouth, among others. ${ }^{40}$ The TEMPO trial noted a significant dropout rate among tolvaptan users due to similar symptoms. ${ }^{33}$ More concerning, the incidence of elevated transaminases in the TEMPO trial was over twice the rate seen in the placebo group, prompting an FDA warning regarding the use of tolvaptan and potential liver injury. ${ }^{41}$ Worth noting, the TEMPO tolvaptan recipients received higher doses, on average, than those used in SIADH trials. Patients beginning 
Table I Summary of characteristics, key end points and net treatment effect of Phase 3 trials of lixivaptan

\begin{tabular}{|c|c|c|c|c|c|c|}
\hline & \multicolumn{2}{|c|}{ BALANCE ${ }^{44,50}$} & \multicolumn{2}{|c|}{ LIBRA $^{44,47}$} & \multicolumn{2}{|c|}{ HARMONY44,48 } \\
\hline & Placebo & Lixivaptan & Placebo & Lixivaptan & Placebo & Lixivaptan \\
\hline Number of subjects & 329 & 323 & 52 & 54 & 52 & 154 \\
\hline Age (years) & 64.7 & 64.9 & 65.2 & 66.4 & 62.7 & 66.6 \\
\hline$\%$ Subjects on fluid restriction - baseline & $62.6 \%$ & $65.0 \%$ & $65.4 \%$ & $37.0 \%$ & $\mathrm{II} .5 \%$ & $16.9 \%$ \\
\hline Initial dose lixivaptan (mg) & N/A & 50 & N/A & 50 & N/A & $25 \mathrm{mg}$ \\
\hline Mean baseline $\mathrm{Na}(\mathrm{mmol} / \mathrm{L})$ & 132.6 & 132.9 & 126.1 & 127.6 & 131.6 & 131.5 \\
\hline Mean $\mathrm{Na} \Delta$ day 7 (mmol/L) & 1.3 & 2.5 & 4.5 & 6.7 & 0.6 & 3.0 \\
\hline Mean $\mathrm{Na} \Delta$ by trial end $(\mathrm{mmol} / \mathrm{L})$ & 1.9 & 2.6 & 4.7 & 6.6 & 1.8 & 3.3 \\
\hline$\%$ of subjects with normalized $\mathrm{Na}$ day 7 & $24.3 \%$ & $30.1 \%$ & $23.1 \%$ & $44.4 \%$ & $12.2 \%$ & $39.4 \%$ \\
\hline Net treatment effect day 7 (mmol/L) & 1.2 & & 2.2 & & 2.4 & \\
\hline
\end{tabular}

Abbreviations: N/A, not applicable; $\Delta$, change.

therapy for SIADH or reinstituting therapy with tolvaptan must be monitored in a hospitalized setting. Both medications are associated with significant expense, and both carry the obvious risk of overly rapid sodium correction, reported in up to $9 \%$ of patients using conivaptan. ${ }^{39}$ This leaves a therapeutic opportunity for a vasopressin antagonist that is able to be initiated in outpatients, requires less frequent monitoring at startup, minimizes drug-drug interactions, and remains broadly effective in euvolemic and hypervolemic hyponatremia.

\section{Lixivaptan pharmacology}

Lixivaptan is a newer, nonpeptide, once-daily oral V2receptor-specific antagonist. Initial in-vitro studies demonstrated significant V2-receptor specificity over the V1a receptor in a mouse fibroblast cell line expressing human vasopressin receptors. ${ }^{42}$ An early trial in patients with chronic heart failure demonstrated maximum urinary output at 2 hours and significant increased solute free water clearance up to 6 hours following administration. ${ }^{43}$ Aquaporin-2 excretion was noted at up to 8 hours in a dose-dependent relationship. Peak serum concentrations occur within 1-2 hours of ingestion, and steady state concentration is achieved in 2-6 days following chronic use. ${ }^{44}$ The half-life of lixivaptan is approximately 11 hours, and metabolism is via the cytochrome P450 system (primarily CYP3A4) with fecal elimination. ${ }^{44,45}$ As with any drug metabolized via the cytochrome P450 system, there is potential for drug-drug interactions, as seen with other vaptans.

\section{Phase 3 clinical trials - BALANCE, LIBRA, HARMONY}

In documents submitted to the FDA, the manufacturer reported a total of 1,673 unique subjects receiving lixivaptan in all Phase 1, 2, and 3 trials. ${ }^{46}$ Three larger, multicenter, international, double-blinded randomized controlled trials have been performed to study lixivaptan in euvolemic hyponatremia and in acute decompensated heart failure. ${ }^{47-49}$ The LIBRA and HARMONY trials evaluated the effect of lixivaptan on SIADH in the inpatient and outpatient settings, respectively, while the BALANCE trial evaluated the drug in congestive heart failure. All three studies used the primary endpoint of serum sodium level following 7 days of drug exposure. Secondary endpoints differed slightly. Design, major attributes, and results are listed for all three trials in Table 1.

\section{BALANCE trial - hypervolemic hyponatremia}

The BALANCE trial is a randomized, double-blind, placebocontrolled trial that was designed to evaluate the effect of lixivaptan in acute decompensated heart failure. ${ }^{49,50}$ Subjects were enrolled based on hospitalization for worsening chronic congestive heart failure, serum sodium level between 120 and $135 \mathrm{mEq} / \mathrm{L}$, and evidence of volume overload as determined by prespecified clinical criteria. Patients were required to meet serum sodium entry criteria with a single measurement within 48 hours of admission to be enrolled. The study was performed between 2008 and 2010 and randomized a total of 652 patients to either lixivaptan $(\mathrm{n}=323)$ or placebo $(\mathrm{n}=329)$. The primary endpoint was change from baseline serum sodium at day 7 of therapy. Secondary endpoints included: (1) time averaged daily serum sodium area under the curve at day 60 ; (2) change from baseline to day 28, in time to complete the Trail Making Test Part B (a neuropsychological test of visual attention and task switching); (3) percentage of subjects with worsening of hyponatremia at any time during the treatment period; (4) percentage of subjects achieving normal serum sodium at days 7 and 60; and (5) days alive and out of the hospital for cardiovascular causes during the double blind on-therapy period. All patients received an initial dose of $50 \mathrm{mg}$ of lixivaptan, with providers instructed to titrate dosing based on 8- and 24-hour repeat 
serum sodium measures, up to a maximum of $100 \mathrm{mg}$ daily. In the final protocol, fluid restriction was discouraged in the first 72 hours, but originally, patients were to be enrolled with hyponatremia, despite baseline fluid restriction.

The lixivaptan group demonstrated a statistically significant but modest difference in the primary endpoint - change in serum sodium at day 7 of $2.5 \mathrm{mEq} / \mathrm{L}$ versus $1.3 \mathrm{mEq} / \mathrm{L}$ in the placebo group $(P=0.001)$, a delta of $1.2 \mathrm{mEq} / \mathrm{L}$ between the two groups. In subsequent comments to the FDA, the sponsor suggested the dose titration scheme may not have been aggressive enough and a higher target sodium may have shown improved separation between groups. Also, the lack of fluid restriction in the first 72 hours, similar to the SALT trials, may also have moderated the effect of lixivaptan. Statistically significant differences in secondary endpoints were similarly modest, and there was no difference in the Trail Making Test Part B. Of note, the only hard clinical endpoint in any of the three trials - days alive and out of the hospital at 60 days, was not different between the two groups.

Adverse events were overall similar in both groups; however, there was an unusually high number of deaths early on in the lixivaptan group for unclear reasons - 15 in the first 10 days of treatment versus four in the placebo group. This prompted the data safety monitoring committee to deliver a letter urging termination of the trial as soon as possible.

\section{LIBRA and HARMONY - euvolemic hyponatremia}

The LIBRA and HARMONY trials were conducted as randomized, double-blind, placebo-controlled trials, designed to evaluate the effects of lixivaptan in euvolemic hyponatremia - specifically SIADH. ${ }^{47,48}$ The trials differed in that LIBRA, like prior studies of vasopressin antagonists, involved in-hospital initiation and monitoring, while HARMONY, uniquely, involved a short monitored initiation period ( 8 hours) followed by outpatient titration.

The LIBRA trial randomized 106 patients to receive either placebo $(n=52)$ versus lixivaptan $(n=54)$, initially at $50 \mathrm{mg}$ daily, titratable to $100 \mathrm{mg}$ daily. Inclusion criteria included a serum sodium $<130 \mathrm{mmol} / \mathrm{L}$ and no overt signs of severe hyponatremia (eg, seizure or coma). Notably, patients with NYHA (New York Heart Association) Class 3 or 4 heart failure were excluded, as were patients with cirrhosis and those with hypovolemic hyponatremia. Both groups were generally similar, with the notable exception of percentage of patients on fluid restriction at trial beginning. Of the placebo patients, $65.4 \%$ began the trial on fluid restriction compared with only $37 \%$ in the lixivaptan group. Though the authors report there was no significant changes in fluid restriction percentage throughout the trial, investigators were allowed to add fluid restriction to pharmacologic therapy after 72 hours of study drug.

Primary and secondary endpoints were generally similar to the BALANCE trial with change in serum sodium at 7 days being the primary endpoint. Differences in secondary endpoints included the addition of "percentage of patients requiring fluid restriction" and a lack of hard clinical endpoints. At the end of 7 days, the least squared mean change from baseline in the lixivaptan group was 6.7 versus $4.5 \mathrm{mmol} / \mathrm{L}$ in the placebo group - a treatment effect of $2.2 \mathrm{mmol} / \mathrm{L}$. The percentage of patients with normal serum sodium at 7 days was roughly double in the lixivaptan group compared with control (44.4\% versus $23.1 \%$ ). Differences in the Trail Making Test Part B were, again, nonsignificant between the two groups.

The HARMONY trial was similar to both BALANCE and LIBRA, with the notable difference that patients were enrolled from a variety of settings that included hospitalized patients, physician offices, long-term care facilities, and others. Overall, $83 \%$ of subjects were not inpatients at time of enrollment. The dose titration monitoring plan was different than any prior Phase 3 trial of vasopressin antagonists. Subjects were administered study drug in a monitored setting, and sodium was rechecked 8 hours later using a point of care sodium monitor. Subjects with a change in serum sodium of $<8 \mathrm{mmol} / \mathrm{L}$ were allowed to leave the monitored setting to return for checks at 24 and 48 hours, while those with a change of $\geq 8 \mathrm{mmol} / \mathrm{L}$ were kept for further observation and serial measurements. Other differences from the previously noted lixivaptan trials included a longer duration of treatment -8 weeks with a follow-on observation phase to 24 weeks total, a lower starting dose of $25 \mathrm{mg}$, and a higher mean sodium of $131 \mathrm{mmol} / \mathrm{L}$ at baseline compared with LIBRA.

A total of 206 patients were randomized in a 3:1 fashion favoring the lixivaptan group. Again, the primary endpoint showed significant difference in serum sodium at 7 days favoring lixivaptan, with a mean improvement of 3.2 versus $0.8 \mathrm{mmol} / \mathrm{L}$ for a treatment effect of $2.4 \mathrm{mmol} / \mathrm{L}$. The percentage of patients on fluid restriction in this trial was much lower and more balanced at outset, with $12 \%$ in the placebo group and $17 \%$ in the lixivaptan group. Significant increases occurred in both groups, but the largest increase came in the placebo group, which at one point had nearly half the group on fluid restriction versus a maximum of $27 \%$ at any point in the lixivaptan group. 
Taken as a group, the Phase 3 clinical trials all met the primary endpoint of statistically significant increased serum sodium versus placebo within 7 days. However, effects in all three trials were modest, with a treatment effect of 1.2, 2.2, and $2.4 \mathrm{mmol} / \mathrm{L}$ for BALANCE, LIBRA, and HARMONY respectively. For comparison, in the SALT trials, effect size was approximately $5 \mathrm{mmol} / \mathrm{L}$ different between placebo and drug groups. ${ }^{31}$

Secondary endpoints were achieved in similar fashion among the trials. Each trial demonstrated a superior number of patients achieving a normal serum sodium at 7 days, and each demonstrated a superior area under the curve of serum sodium at trial end dates. The effect of the difference in fluid restriction, notably in LIBRA, is difficult to account for. Given that the groups were otherwise similar, the much lower percentage of patients initially on fluid restriction in the lixivaptan group may suggest those patients, as a group, had better free water clearance versus the control group, a potential confounder as the authors acknowledge. Tests of cognitive function were also ambiguous. The Trail Making Test Part B scores were not significantly different between placebo and lixivaptan groups, but there was statistically significant improvement in scores within the lixivaptan groups from trial beginning to end. This may suggest more about hyponatremia and cognitive effect than the effect of the study drug. In fact, FDA analysis of the BALANCE trial data suggested a statistically significant difference favoring the placebo group, making any conclusions on cognitive function difficult to draw. ${ }^{50}$ Other clinical endpoints were not assessed. For example, in LIBRA, many common symptoms associated with hyponatremia were described at baseline but not formally assessed for improvement as the trial progressed. While these studies were not primarily designed to evaluate improvement in symptoms such as nausea, fatigue, for example, with correction of serum sodium, this would have been a welcome analysis.

\section{FDA ruling}

On September 13, 2012, the Cardiovascular and Renal Drugs Advisory Committee of the FDA voted unanimously against recommending lixivaptan for treatment of hypervolemic hyponatremia and split five to three against recommending the drug for treatment of euvolemic hyponatremia. The internal analysis released prior to the committee meeting suggested approval for the treatment of SIADH based on statistically significant improvements in serum sodium, which had been accepted by the agency as basis for the approval of both conivaptan and tolvaptan. ${ }^{50}$ However, the FDA analysis raised concerns regarding the small treatment effect size in the BALANCE trial relating to decompensated heart failure, especially in light of the high mortality rate in the first 5 days of medication administration.

The formal committee meeting echoed similar sentiments. Regarding use in hypervolemic hyponatremia, reviewers cited concerns regarding treatment effect size and clinical meaningfulness of surrogate endpoints such as serum sodium change in the lixivaptan group. ${ }^{51}$ Mortality concerns were expressed by many, but not all members, and most agreed that further studies would be warranted in this group to address safety concerns, consider a more aggressive sodium target to allow for better separation, and if possible, involve more useful clinical endpoints.

Regarding use in euvolemic hyponatremia, the committee was split on use, though themes were similar. Most agreed clinical utility, aside from possible reduction in fluid restriction, was not demonstrated in these trials. That said, reviewers that voted for use felt that serum sodium as a surrogate marker had been a reasonable standard in prior approvals and could not, in fairness, be abandoned in considering lixivaptan. Concerns were also raised regarding the logistical ability to initiate outpatient administration of lixivaptan and replicate a similar scheme as used in the trial. If this proved impossible, then lixivaptan would effectively be limited to inpatient initiation, as are other agents in the class.

\section{Future studies}

The class of vasopressin receptor antagonists has held great promise based on clinical trials, but most have relied on the surrogate endpoint of rise in serum sodium. Many have noted previously the lack of consensus on the appropriate use of these medications by the clinician. ${ }^{52,53}$ As noted in the overview section, hyponatremia appears to have broad deleterious effects, even in previously considered asymptomatic hyponatremia. Trials of vaptans assessing hard clinical outcomes, such as EVEREST (Efficacy of Vasopressin Antagonism in Heart Failure Outcome Study with Tolvaptan) for heart failure patients, have not shown a long-term mortality benefit and nor do data exist for mortality benefit in patients with cirrhosis. ${ }^{38}$ It seems clear that vaptans, when used in a similar fashion to published trials, are generally safe and can reliably increase serum sodium; however, data on meaningful clinical improvement is lacking, as reflected in the FDA committee ruling on lixivaptan.

Ideally, future trials of lixivaptan would include more clinically meaningful endpoints encompassing both hard 
outcomes such as mortality and days out of hospital as well as symptomatic/patient-centered endpoints to evaluate symptom improvement. In addition, as suggested by the sponsor, the modest differences seen in serum sodium may have been partially a result of a conservative dose titration scheme. HARMONY, for example, used a low starting dose specifically to avoid overcorrection in the outpatient setting. The need to show a more significant improvement in serum sodium must be balanced in light of the mortality data seen in BALANCE; though most adverse events were more numerous in the control groups in the three trials.

Regarding the practicality of administering lixivaptan, the idea of outpatient initiation is a compelling positive, but only if the economic benefit of an avoided hospitalization or other costs are abrogated by use of the drug. Furthermore, safety concerns regarding this strategy require more data and the development of infrastructure in the outpatient setting to support this protocol. This would allow a more compelling case for lixivaptan. Certainly, the addition of a competing oral vasopressin antagonist could only benefit patients who currently find outpatient therapy cost-prohibitive.

In conclusion, it appears that lixivaptan is an effective means to raise serum sodium in both the heart failure population and in patients with euvolemic hyponatremia from SIADH. Questions remain about the overall clinical benefit and practicality of an outpatient titration regimen, which warrant further studies.

\section{Disclosure}

The authors report no conflicts of interest in this work.

\section{References}

1. Upadhyay A, Jaber BL, Madias NE. Incidence and prevalence of hyponatremia. Am J Med. 2006;21:70-76.

2. Hawkins RC. Age and gender as risk factors for hyponatremia and hypernatremia. Clin Chim Acta. 2003;337:169-172.

3. Liamis G, Rodenburg EM, Hofman A, Zietse R, Stricker BH, Hoorn EJ. Electrolyte disorders in community subject: prevalence and risk factors. Am J Med. 2013;126(3):256-263.

4. Adrogue HJ, Madias NE. Hyponatremia. N Engl J Med. 2000;342(21): 1581-1589.

5. Frase CL, Arieff AI. Epidemiology, pathophysiology, and management of hyponatremia encephalopathy. Am J Med. 1997;102:67-77.

6. Hoorn EJ, Rivadeneira F, van Meurs JB, et al. Mild hyponatremia as a risk factor for fractures: the Rotterdam Study. J Bone Miner Res. 2011;26(8): 1822-1828.

7. Waikar SS, Mount DB, Curhan GC. Mortality after hospitalization with mild, moderate, and severe hyponatremia. 2009;122:857-865.

8. Shea AM, Hammill BG, Curtis LH, Szczech LA, Schulman KA. Medical costs of abnormal serum sodium levels. J Am Soc Nephrol. 2008;19: 764-770.

9. Rose BD, Post TW. Clinical Physiology of Acid-Base and Electrolyte Disorders. 5th ed. New York: McGraw Hill; 2001.

10. Schrier RW, Berl T, Anderson RJ. Osmotic and nonosmotic control of vasopressin release. Am J Physiol. 1979;236:F321-F332.
11. Koshimizu T, Nakamura K, Egashira N, Hiroyama M, Nonoguchi H, Tanoue A. Vasopressin V1a and V1b receptors: from molecules to physiological systems. Physiol Rev. 2012;92:1813-1864.

12. Nielsen S, Kwon TH, Christensen BM, Promeneur D, Frøkiaer J, Marples D. Physiology and pathophysiology of renal aquaporins. J Am Soc Nephrol. 1999;10:647-663.

13. Kang SH, Kim HW, Lee SY, et al. Is the sodium level per se related to mortality in hospitalized patients with severe hyponatremia? Clin Nephrol. 2012;77(3):182-187.

14. Hoorn EJ, Lindemans J, Zietse R. Development of severe hyponatraemia in hospitalized patients: treatment-related risk factors and inadequate management. Nephrol Dial Transplant. 2006;21:70-76.

15. Arief AI, Llach F, Massry S. Neurological manifestation and morbidity of hyponatremia: correlation with brain water and electrolytes. Medicine. 1976;55(2):121-129.

16. Schrier R, Shweta B. Diagnosis and management of hyponatremia in acute illness. Curr Opin Crit Care. 2008;14(6):627-634.

17. Renneboog B, Musch W, Vandemergel X, Manto MU, Decaux G. Mild chronic hyponatremia is associated with falls, unsteadiness, and attention deficits. 2006. Am J Med. 2006;119(1):71. e1-e8.

18. Verbalis JG, Barsony J, Sugimura Y, et al. Hyponatremia-induced osteoporosis. J Bone Miner Res. 2010;25;(3):554-563.

19. Wald R, Jaber BL, Price LL, Upadhyay A, Madias NE. Impact of hospital-associated hyponatremia on selected outcomes. Arch Intern Med. 2010;170(3):294-302.

20. Klein L, O'Connor CM, Leimberger JD, et al. Lower serum sodium is associated with increased short-term mortality in hospitalized patients with worsening heart failure: results from the Outcomes of a Prospective Trial of Intravenous Milrinone for Exacerbations of Chronic Heart Failure (OPTIME-CHF) study. Circulation. 2005;111(19): 2454-2460.

21. Jenq CC, Tsai MH, Tian YC, et al. Serum sodium predicts prognosis in critically ill cirrhotic patients. J Clin Gastroenterol. 2010;44(3): 220-226.

22. Boscoe A, Paramore C, Verbalis JG. Cost of illness of hyponatremia in the United States. Cost Eff Resour Alloc. 2006;4:10.

23. Callahan MA, Do HT, Caplan DW, Yoon-Flannery K. Economic impact of hyponatremia in hospitalized patients: a retrospective cohort study. Postgrad Med. 2009;121(2):186-191.

24. Zilberberg MD, Exuzides A, Spalding J, et al. Epidemiology, clinical and economic outcomes of admission hyponatremia among hospitalized patients. Curr Med Res Opin. 2008;24(6):1601-1608.

25. Schrier RW, Sharma S, Shchekochikhin D. Hyponatraemia: more than just a marker of disease severity? Nat Rev Nephrol. 2013;9(1): $37-50$.

26. Soupart A, Coffernils M, Couturier B, Gankam-Kengne F, Decaux G. Efficacy and tolerance of urea compared with vaptans for long-term treatment of patients with SIADH. Clin J Am Soc Nephrol. 2012;7(5): 742-747.

27. Shchekochikhin D, Tkachenko O, Schrier RW. Hyponatremia: an update on current pharmacotherapy. Expert Opin Pharmacother. 2013;14(6): $747-755$.

28. US Food and Drug Association. Drug Approval Package. Conivaptan. Available from: http://www.accessdata.fda.gov/drugsatfda_docs/ nda/2007/022016_vaprisol_toc.cfm. Accessed April 28, 2013.

29. US Food and Drug Association. Approved Drug Products. Tolvaptan. Available from: http://www.accessdata.fda.gov/scripts/cder/drugsatfda/ index.cfm?fuseaction=Search. Accessed April 28, 2013.

30. Quittnat F, Gross P. Vaptans and the treatment of water-retaining disorders. Semin Nephrol. 2006;26(3):234-243.

31. Schrier RW, Gross P, Gheorghiade M. Tolvaptan, a selective oral vasopressin V2-receptor antagonist, for hyponatremia. $N$ Engl J Med. 2006;355(20):2099-2112.

32. Udelson JE, Smith WB, Hendrix GH, et al. Acute hemodynamic effects of conivaptan, a dual $\mathrm{V}(1 \mathrm{~A})$ and $\mathrm{V}(2)$ vasopressin receptor antagonist, in patients with advanced heart failure. Circulation. 2001;104(20): $2417-2423$. 
33. Torres VE, Chapman AB, Devuyst $\mathrm{O}$, et al. Tolvaptan in patients with autosomal dominant polycystic kidney disease. $N$ Engl J Med. 2012;367(25):2407-2418.

34. Zeltser D, Rosansky S, van Rensburg H, Verbalis JG, Smith N. Assessment of the efficacy and safety of intravenous conivaptan in euvolemic and hypervolemic hyponatremia. Am J Nephrol. 2007;27(5): 447-457.

35. Ghali JK, Koren MJ, Taylor JR, et al. Efficacy and safety of oral conivaptan: a $\mathrm{V} 1 \mathrm{~A} / \mathrm{V} 2$ vasopressin receptor antagonist, assessed in a randomized, placebo-controlled trial in patients with euvolemic or hypervolemic hyponatremia. J Clin Endocrinol Metab. 2006;91(6):2145-2152.

36. Annane D, Decaux G, Smith N. Efficacy and safety of oral conivaptan, a vasopressin-receptor antagonist, evaluated in a randomized, controlled trial in patients with euvolemic or hypervolemic hyponatremia. Am J Med Sci. 2009;337(1):28-36.

37. Gheorghiade M, Gattis WA, O'Connor CM, et al. Effects of tolvaptan, a vasopressin antagonist, in patients hospitalized with worsening heart failure: a randomized controlled trial. JAMA. 2004;291(16):1963-1971.

38. Konstam MA, Gheorghiade M, Burnett JC Jr. Effects of oral tolvaptan in patients hospitalized for worsening heart failure: the EVEREST Outcome Trial. JAMA. 2007;297(12):1319-1331.

39. Li-Ng M, Verbalis JG. Conivaptan: evidence supporting its therapeutic use in hyponatremia. Core Evid. 2010;4:83-92.

40. Berl T, Quittnat-Pelletier F, Verbalis JG. Oral tolvaptan is safe and effective in chronic hyponatremia. J Am Soc Nephrol. 2010;21(4): $705-712$.

41. US Food and Drug Association. MedWatch: The FDA Safety Information and Adverse Event Reporting Program. Samsca (tolvaptan): Drug Warning - Potential Risk of Liver Injury. Available from: http://www. fda.gov/Safety/MedWatch/SafetyInformation/SafetyAlertsforHuman MedicalProducts/ucm336669.htm. Accessed April 28, 2013.

42. Albright JD, Reich MF, Delos Santos EG, et al. 5-Fluoro-2-methyl$\mathrm{N}$-[4-(5H-pyrrolo[2,1-c]-[1,4]benzodiazepin-10(11H)-ylcarbonyl)3-chlorophenyl]benzamide (VPA-985): an orally active arginine vasopressin antagonist with selectivity for V2 receptors. $J$ Med Chem. 1998;41(14):2442-2444.

43. Abraham WT, Shamshirsaz AA, McFann K, Oren RM, Schrier RW. Aquaretic effect of lixivaptan, an oral, non-peptide, selective V2 receptor vasopressin antagonist, in New York Heart Association functional class II and III chronic heart failure patients. J Am Coll Cardiol. 2006;47(8):1615-1621.
44. Cardiokine Biopharma, LLC. Advisory Committee Briefing Document. Silver Spring, MD: US Food and Drug Administration; 2012. Available from: http://www.fda.gov/downloads/AdvisoryCommittees/ CommitteesMeetingMaterials/Drugs/CardiovascularandRenalDrugs AdvisoryCommittee/UCM318869.pdf. Accessed April 28, 2013.

45. Ku E, Nobakht N, Campese VM. Lixivaptan: a novel vasopressin receptor antagonist. Expert Opin Investig Drugs. 2009;18(5):657-662.

46. Cardiokine Biopharma, LLC. Cardiokine Slides, Lixivaptan, for the September 13, 2012 Meeting of the Cardiovascular and Renal Drugs Advisory Committee. Available from: http://www.fda.gov/downloads/ AdvisoryCommittees/CommitteesMeetingMaterials/Drugs/CardiovascularandRenalDrugsAdvisoryCommittee/UCM320601.pdf.

47. Abraham WT, Hensen J, Gross PA, et al. Lixivaptan safely and effectively corrects serum sodium concentrations in hospitalized patients with euvolemic hyponatremia. Kidney Int. 2012;82(11):1223-1230.

48. Abraham WT, Decaux G, Josiassen RC. Oral lixivaptan effectively increases serum sodium concentrations in outpatients with euvolemic hyponatremia. Kidney Int. 2012;82(11):1215-1222.

49. Abraham WT, Aranda JM, Boehmer JP. Rationale and design of the treatment of hyponatremia based on lixivaptan in NYHA class III/IV cardiac patient evaluation (THE BALANCE) study. Clin Transl Sci. 2010;3(5):249-253.

50. US Food and Drug Association. FDA Briefing Document for the Cardiovascular and Renal Drugs Advisory (CRDAC). Silver Spring, MD: US Food and Drug Administration; 2012. Available from: http://www.fda. gov/downloads/AdvisoryCommittees/CommitteesMeetingMaterials/ Drugs/CardiovascularandRenalDrugs AdvisoryCommittee/ UCM318867.pdf. Accessed April 28, 2013.

51. US Food and Drug Association. Transcript of the FDA center for drug evaluation and research cardiovascular and renal drugs advisory committee Thursday, September 13, 2012 8:00 am to 12:00 pm Morning Session. Available from: http://www.fda.gov/downloads/ AdvisoryCommittees/CommitteesMeetingMaterials/Drugs/ CardiovascularandRenalDrugsAdvisoryCommittee/UCM345074.pdf. Accessed April 28, 2013.

52. Borne RT, Krantz MJ. Lixivaptan for hyponatremia - the numbers game. JAMA. 2012;308(22):2345-2346.

53. Gross PA, Wagner A, Decaux G. Vaptans are not the mainstay of treatment in hyponatremia: perhaps not yet. Kidney Int. 2011;80: 594-600.
Core Evidence

\section{Publish your work in this journal}

Core Evidence is an international, peer-reviewed open-access journal evaluating the evidence underlying the potential place in therapy of drugs throughout their development lifecycle from preclinical to postlaunch. The focus of each review is to evaluate the case for a new drug or class in outcome terms in specific indications and patient groups.

\section{Dovepress}

The manuscript management system is completely online and includes a very quick and fair peer-review system, which is all easy to use. Visit http://www.dovepress.com/testimonials.php to read real quotes from published authors. 